P\&A Año 2, N. ${ }^{\circ 3}$

julio-diciembre 2017

pp. [17]-30

\title{
Resumen
}

El avance científico y tecnológico alcanzado en el siglo XXI permite reflexionar, como docentes de las diversas carreras que existen en el mundo, en especial, el de la Carrera de Arquitectura; sobre las nuevas corrientes de la Pedagogía contemporánea surgidas desde hace cerca de veinte años, en relación a la enseñanza-aprendizaje del Diseño Arquitectónico, como es la Neuroarquitectura. La aplicación de esta nueva corriente potenciaría el diseño arquitectónico en su práctica y el conocimiento de la conducta humana durante la etapa de aprendizaje, por los estudiantes de arquitectura. Esto permitiría a docentes y alumnos conocer y desarrollar las bondades ocultas del mismo y su relación con la Creatividad, insumo cualitativo de toda producción arquitectónica.

Palabras claves: Neuroarquitectura y creatividad, aprendizaje, diseño arquitectónico, proceso creativo

\section{Neuroarquitectura y creatividad en el aprendizaje del diseño arquitectónico*}

\author{
Neuroarchitecture, creativity and the learning of architectonic design \\ Dr. Laurente Jesús Gutiérrez Talledo**
}

Recibido: 29 de abril de 2017

Aceptado: 23 de junio de 2017

\begin{abstract}
The scientific and technological progress achieved in the 21st century, allows us to reflect as professors of the various careers that exist in the world, especially, the Career of Architecture; on the new currents of Contemporary Pedagogy which have emerged for about twenty years, in relation to the teaching-learning of Architectural Design, as it is the Neuroarchitecture. The application of this new current would enhance the architectural design in its practice and knowledge of human behavior during the learning stage specifically, by architecture students. This would allow professors and students to know and develop the hidden benefits of it and its relationship with Creativity, qualitative input of any architectural production.
\end{abstract}

Keywords: Neuroarchitecture and creativity, learning, architectural design, creative process

\footnotetext{
* El presente artículo es una ampliación de una de las variables de la investigación realizada por el autor y presentada como tesis doctoral titulada: Estrategias y Técnicas para el Desarrollo de la Creatividad en el Proceso de Diseño Arquitectónico en los alumnos de los Talleres de Diseño Arquitectónico de la FAU-URP (2016), para obtener el grado de doctor en Educación.

** Arquitecto por la Universidad Nacional de Ingeniería, doctor en Educación y magíster en Arquitectura y Urbanismo por la Universidad Nacional Federico Villarreal, Especialista en Didáctica Universitaria por la Universidad Ricardo Palma y profesor ordinario de la Facultad de Arquitectura y Urbanismo de la Universidad Ricardo Palma. Lima-Perú.
} 
Así pues, el habitar sería en cada caso el fin que preside todo construir.

Martin Heidegger

\section{Introducción}

En principio, la intención de este artículo es pedagógico, pero al mismo tiempo se dirige hacia la búsqueda de la esencia de la enseñanza-aprendizaje de la arquitectura en general y del aprendizaje del diseño arquitectónico en particular, en las actuales escuelas de arquitectura del país; en su sentido más original y universal con el fin de encontrar en el análisis de la neurociencia y de la psicología, y su relación con la creatividad en el aprendizaje del diseño arquitectónico, el sustento epistemológico o elemento básico del quehacer del diseño en arquitectura en su etapa fundamental. Esto permite planear la reflexión que debe preceder a cualquier currículo y qué debe plantearse en cualquier facultad con la claridad que le permita, a estudiantes y profesores enmarcar, fundamentar su actuación de enseñanza-aprendizaje en el diseño arquitectónico como tarea fundamental y específica, en el diseño creativo del espacio y su habitabilidad.
Con la premisa anteriormente expuesta, se trata de establecer una relación dialógica entre lo pedagógico, lo académico de la enseñanza de la arquitectura y su relación con las nuevas teorías del diseño arquitectónico con un enfoque neurocientífico, en el aprendizaje del diseño arquitectónico, específicamente; con una formación más humana y científica para la producción de los espacios habitables en armonía con el bienestar y el confort del ser humano como protagonista del mismo y su relación con el entorno y el Medio Ambiente en que se desarrolla. Existen teorías contemporáneas que relacionan el estudio del comportamiento y/o conducta del usuario como reacción fisio-psicológica ante la acción o influencia del espacio arquitectónico; como es la neuroarquitectura en el diseño de espacios habitables. Aquí se trata de la aplicación de una teoría científica que nace de la neurociencia y de la neuropedagogía, las que se ocupan del estudio científico del funcionamiento del cerebro; en este caso, en la etapa de diseño arquitectónico por parte del estudiante.

Esto conlleva a una reflexión y revisión del diseño curricular de la carrera de arquitectu-

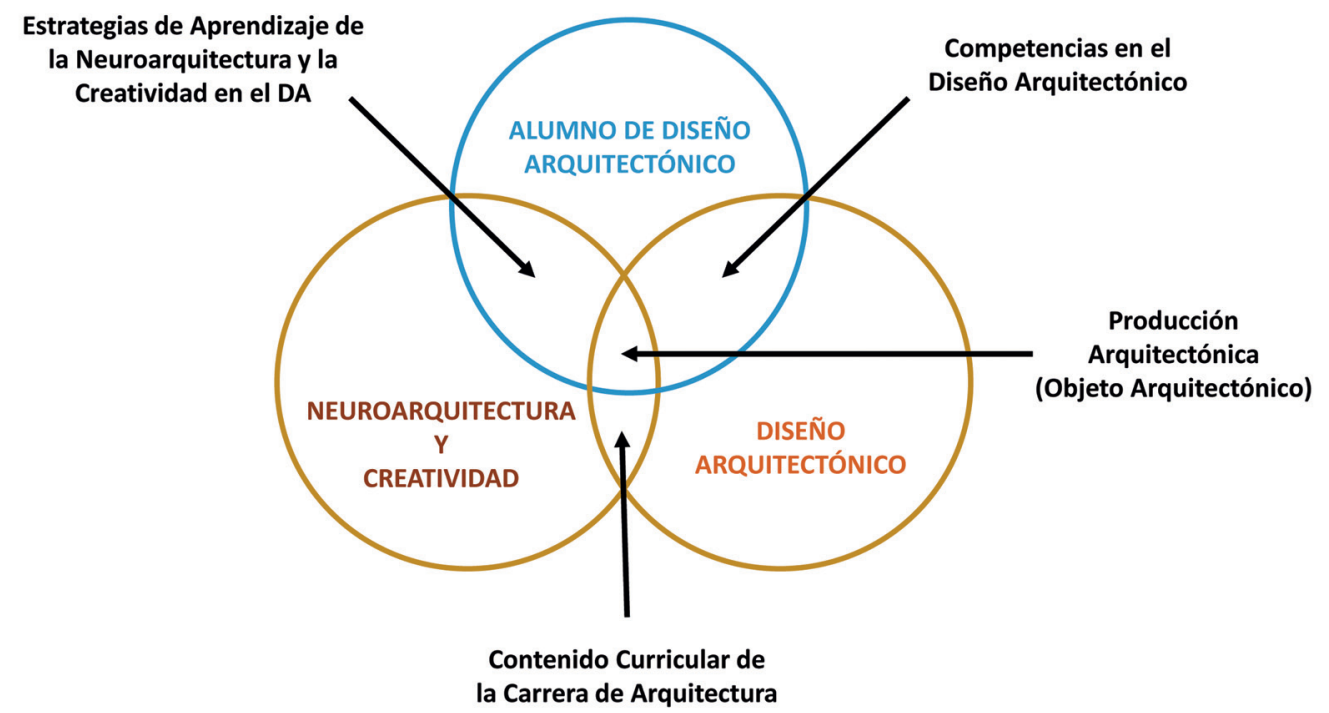

Figura 1. Esquema conceptual de la investigación. [Material gráfico de apoyo, elaboración propia, 2017]. 
ra para un cambio en el diseño y contenidos de las competencias, habilidades y actitudes del estudiante y a una integración pedagógica y académica de los cursos básicos y/o complementarios que apoyan al Taller de diseño, como curso formativo estructural.

\section{La neuroarquitectura y el diseño arquitectónico}

Uno de los componentes principales del diseño arquitectónico es el diseño del Espacio arquitectónico, si entendemos en su significado etimológico, que provienen de los vocablos latinos spatium y architectonicus, respectivamente; es decir, la noción de espacio arquitectónico hace referencia al lugar cuya producción es el objeto de la arquitectura y el concepto de espacio arquitectónico, que es diverso, se encuentra en permanente revisión por parte de expertos en la materia (Pérez \& Gardey, 2014).

Al respecto Moreno (2008) considera el espacio arquitectónico como el lugar donde habita el hombre y por lo tanto se podría definir, para este caso, al diseño arquitectónico como la ciencia del diseño del espacio habitable o con habitabilidad, entendiéndose como tal, según Castro (1999), citado en Villagrán. (2017):

al concepto que se refiere a la satisfacción que uno obtiene en un determinado escenario o grupo de escenarios; es el atributo de los espacios construidos de satisfacer las necesidades objetivas y subjetivas de los individuos y grupos que las ocupan, es decir, las esferas psíquicas y sociales de la existencia estable que podría equipararse a las cualidades medioambientales que permitan el sano desarrollo físico, biológico, psicológico y social de la persona. (p. 32)

Y si de espacio arquitectónico se trata, nos referimos explícitamente al espacio interior arquitectónico, que es el que principalmente se diseña en forma académica en las escuelas de arquitectura. Por lo general, según Muñoz (2012):

Los ensayos sobre el espacio arquitectónico se inciden tácita y expresamente en el espacio interior como cosa principal. $Y$ es porque éste es primigenio. Lo que está primero es lo que necesitams antes que nada, lo que primero hacemos por la necesidad natural que tenemos de protección, de techo, de interioridad, de privacidad. (p. 3)

El presente artículo no escapa a esa especificidad y es ese el espacio, conceptualizado, diseñado y construido; el que influye directamente en el estado emocional y comportamiento del usuario que lo consume, que lo recorre y usa. Al estudio de la acción a través de la percepción del sujeto del espacio percibido y de la reacción de la imagen captada por el cerebro, produciendo sensaciones y comportamientos diversos del sujeto en su conducta, se le denomina neuroarquitectura.

\section{El cerebro humano y la percepción del espacio}

El espacio percibido otorga sensaciones positivas o negativas en el comportamiento humano. Alison Whitelaw (2012) citada en Elizondo \& Rivera (2014), declara que: "La calidad del ambiente construido puede afectar el desempeño del cerebro; como la experiencia del ambiente donde se encuentran las personas puede influir en su estado emocional y en su comportamiento" (p. 41).

Epstein y Kanwisher (1999), citados también por Elizondo \& Rivera (2014), señalan que:

existe una región del cerebro llamada en inglés Parahippocampal Place Area (PPA); esta región se encuentra en el hipocampo, la región del cerebro que se dedica a procesar nueva información y almacenar las memorias y recuerdos. La PPA se activa únicamente con la percepción de lugares, cada vez que un individuo se encuentra en un determinado lugar o recuerda de manera novedosa este espacio. (p. 42)

Los mismos autores agregan: "que la PPA es más activa cuando las personas observan escenas complejas como paisajes, ciudad, habitaciones con muebles, y estas experiencias son almacenadas dentro de nosotros" (p. 42).

En ese sentido, se puede inferir que la percepción del sujeto del espacio en que participa es gravitante para generar diversas sensaciones -qué dependiendo de la receptividad de su ce- 
rebro- hace que éste sea un espacio habitable que genere confort en sus diversas maneras, que sea adecuado para la función o actividad para lo cual fue destinado; en este caso utilitario, para satisfacer necesidades primarias y/o secundarias del sujeto que percibe.

Las características físicas y formales del espacio creado y percibido, dependerán de generar sensaciones positivas y/o negativas en el sujeto observador que se darán de manera simultánea durante el proceso creativo, y que resultarán del diseño creativo experimental del proyectista como diseñador en una primera fase y como usuario en una segunda fase.

Según Elizondo y Rivera (2014), la ANFA (Academia de Neurociencia de Arquitectura) señala tres factores claves para crear mejores espacios:

El primero, es la continuidad del espaciotiempo. Dentro del cerebro, específicamente en la región del hipocampo, están las neuronas que reaccionan cuando las personas se encuentran en un lugar o espacio específico. Estas neuronas ayudan a fomentar de manera interna una idea o experiencia espacial del mundo exterior a su persona.

El segundo factor clave, es el impacto de la arquitectura en la percepción espacial. Como arquitectos o diseñadores es importante ser sensatos a la hora de diseñar espacios porque cualquier elemento puede ocasionar algún problema para otro habitante, como en el caso del diseño de espacios para personas con problemas psiquiátricos en donde algún elemento difícil de identificar pueden ocasionarles problemas de desubicación, estrés o molestia neural.

Como último factor clave, se tiene la iluminación. Desde aspectos fisiológicos es de vital importancia la luz natural para el cuerpo humano, pues si no se cuenta con una adecuada iluminación natural puede ocasionar estados de ánimo negativos. Por esto es importante que las personas estén expuestas a $2 \mathrm{mil} \mathrm{lu-}$ xes en un promedio de una hora diaria para neutralizar la depresión que se puede generar en las personas por la falta de iluminación natural.

El surgimiento de la neuroarquitectura como ciencia, que resulta de unir los estudios de la neurociencia y de la arquitectura, ha merecido opiniones diversas sobre la consideración de la primera en el diseño de los espacios arquitectónicos y su influencia en el cerebro del sujeto que lo percibe. Las autoras Elizondo \& Rivera (2014), citan las referidas opiniones de Fred Gage (2003), quien presentó este descubrimiento en una convención de arquitectos, en el Instituto Americano de Arquitectura. Y enunció la siguiente idea: "Los cambios en el entorno, cambian el cerebro, por tanto, modifican nuestro comportamiento" (p. 43).

Mientras que Zeisel (2006) sostiene que el reto actual para la arquitectura es intimar con el cerebro, entender cómo funciona y el por qué hay espacios que favorecen ciertos estados de ánimo. El autor indaga en el campo de la neurociencia para describir el impacto de los edificios y de los espacios en la vida de las personas. Se trata de tener un conocimiento preciso del tema, para concebir edificios y espacios en consonancia con el bienestar no sólo físico, sino también mental.

Para Barros (2013) el ser humano se ha percatado del influjo del espacio sobre la psique y que, si esta variable se contempla intencionalmente al momento de construir un inmueble, pueden conseguirse resultados específicos.

Por su parte Sáez (2014) dice:

Aunque la Neuroarquitectura es un concepto bastante novedoso, que los arquitectos tomen en cuenta principios de salud a la hora de diseñar inmuebles, no lo es. Y es lógico que sea así, porque más del $90 \%$ del tiempo que estamos despiertos al día lo pasamos dentro de edificios, y lamentablemente muchos de los cuales no están pensados y construidos para hacernos sentir bien. (p. 2)

De las opiniones expuestas se puede inferir las bondades de la neuroarquitectura en el diseño arquitectónico y la necesidad de contar con ella, para la creación de los espacios habitables, explicados anteriormente. El diseñador representa un doble rol en el proceso 
de diseño como proyectista y como partícipe del espacio a diseñar en forma simultánea durante el acto proyectual; convirtiendo el diseño arquitectónico en una disciplina multidisciplinar con la intervención de la psicología, neurología y la propia arquitectura, que modifica el enfoque de una nueva concepción en la enseñanza y el aprendizaje del diseño arquitectónico en las actuales escuelas de arquitectura.

\section{La Creatividad y el Diseño Arquitectónico. La percepción del espacio construido}

Aún puede considerarse como muy reciente el inicio de las investigaciones y estudios sobre el campo de conocimiento de la creatividad. Las primeras aportaciones de autores como De Bono, Osborn o Torrance datan de la década de los sesenta en adelante, por lo que la aplicación práctica de todo lo hallado a nivel teórico en los centros educativos es todavía insuficiente.

La creatividad es una preocupación central de la cultura contemporánea y ella se extiende a otros campos profesionales. El término creatividad tiene múltiples acepciones y es comprendido de distintas maneras. Hoy día se habla de creatividad en las empresas y se refiere a la habilidad para encontrar nuevas rentabilidades en ambientes hostiles, cambiantes y de gran competitividad. La biología también está hablando de creatividad, pero ella la relaciona con la maravillosa capacidad de los seres vivos de adaptarse a entornos cambiantes e impredecibles. Se postula aquí que la creatividad sería una condición innata de los seres vivos (Fox, 2001).

Respecto al diseño arquitectónico como proceso, el mismo Fox (2009) manifiesta:

que como proceso representa también una forma de actuar, de intervenir y de transformar el mundo. En dicho proceso se concatenan, simultánea o secuencialmente, procedimientos y operaciones con metas y objetivos y, ellos a su vez, con expectativas y visiones de futuro. Esto incluye valores y motivaciones, siempre en la perspectiva de lograr calidad, confiabilidad y concordancia en los resultados. Es parte fundamental de este proceso la visualización de las consecuencias y los impactos en los contextos, sean ellos territorial-espaciales, sociales, económicos, culturales o medioambientales, cuyas implicaciones desbordan y muchas veces superan el accionar del propio diseñador. (p. 5)

Con respecto a la relación entre creatividad y diseño arquitectónico como procesos, Lesbia (2008) dice:

En el proceso de diseño la creatividad juega un papel nuclear porque es expresión de la complejidad del mismo proceso dado que el trabajo es asignado por un cliente o profesor, según sea el caso, pero la persona que interviene debe, desde su propia multidimensionalidad y complejidad; dar respuesta a la propuesta planteada, tomando en consideración todos los elementos que confluyen en esa obra. La creatividad aquí debe obedecer a un proceso interno de auto organización de elementos que lleven a la coherencia en la expresión, siguiendo los principios, normas y leyes del diseño. En este proceso interno hay que darle un amplio espacio al momento de reflexión, al autoencuentro consigo mismo, a la toma de contacto con su internalidad, al silencio interior, para que afloren las ideas y ocupen los espacios de la obra creada. (pp. 7-8)

En ese sentido, la relación entre creatividad y diseño arquitectónico es simbiótico, inherente e interdependiente; sin creatividad no hay proceso de diseño creativo, innovador y original, y sin proceso de diseño creativo no se puede obtener un producto novedoso.

Es de interés en esta investigación ocuparse de la creatividad diseñadora en los talleres de arquitectura y, específicamente, en el diseño arquitectónico. Si bien es cierto, y lo dicen muchos estudios al respecto, que el estudio de la creatividad se ha referido a su origen, a su proceso y producto; lo que se quiere es profundizar su incidencia en el proceso de diseño arquitectónico como herramienta para la investigación en general y para la experimentación en particular de la disyunción entre sujeto y objeto, a lo que denomina Fox (2001) la creatividad experimental; es decir, el tratamiento del sujeto creativo y del producto creado como un todo, inseparables a pesar de su diferente naturaleza, con el pro- 
pósito de dar soluciones a problemas complejos que emanan de establecer o esclarecer la relación sujeto y objeto.

Si se lleva esta visión filosófica y epistemológica de la creatividad experimental a la relación sujeto-creativo y objeto-espacio en el proceso de diseño arquitectónico; se establece una relación interdependiente entre el diseñador y el espacio creado, obteniéndose resultados positivos en cuanto al uso de lugares más agradables al usuario lo cual genera sensaciones positivas en el uso del espacio percibido que conlleva a una adaptación o adecuación y disfrute del confort, sin llegar a alterar fisiológicamente el estado psicológico y físico del sujeto. Es aquí donde es necesario que el sujeto proyectista, se internalice en el proceso de diseño en forma experimental y actúe como sujeto creativo y como sujeto usuario simultáneamente. De lo expuesto se puede reafirmar que de la relación e interdependencia sujeto-objeto se produce una relación interdisciplinaria entre la Arquitectura y la Psicología; es decir, la neuroarquitectura, la ciencia que estudia la influencia del objeto-espacio en las sensaciones y conducta de comportamiento humano del sujeto-usuario.

\section{La psicología del diseño del espacio (interior) arquitectónico}

El espacio, al que nos referimos en este caso, como objeto del proceso de diseño arquitectónico, es el espacio habitado por el sujeto o usuario -en donde el sujeto creador y usuario al mismo tiempo durante el proceso creativo y de diseño arquitectónico- conceptualiza el objeto-espacio, pero este está referido al espacio arquitectónico porque está contenido en la Arquitectura, al respecto Cardoni (2009) dice:

El espacio arquitectónico. Para que podamos comprender la arquitectura desde un punto de vista perceptual tendremos en cuenta aquellas percepciones que se llaman espacio y lugar arquitectónico. Si no está presente esta idea, ya no se considera arquitectura sino escultura, mobiliario, artesanía o diseño industrial. (p. 28)
Así mismo, el autor de la nota considera además los dos espacios de la arquitectura, el espacio exterior que es ilimitado y es aquel en el cual está inserto el objeto construido, en contraposición al espacio interior que se percibe cerrado, limitado y porción del anterior delimitado por la construcción. La experiencia del espacio interior podrá ser modificada de acuerdo a la ubicación de observación que elija el observador. En el espacio exterior, la experiencia reviste otra característica, ya que el observador se ubica al borde desde el cual contempla, más que rodeado por el espacio.

Entonces, podemos decir que la experiencia del espacio interior estará íntimamente vinculada con el mundo de la percepción y su estructura diferencial. Hesselgren (1969) entiende como experiencia del espacio interior al resultado del proceso de percepción e informe de la acción, constituyendo ambos, la esencia de la experiencia en el habitar (p. 30).

Al respecto, Aisa (2012) cita a Pallasma (2006), quien afirma que la experiencia arquitectónica es el "acercarse o enfrentarse a un edificio, más que la percepción formal de una fachada; el acto de entrar y no simplemente del diseño visual de la puerta; mirar al interior o al exterior por una ventana, más que la ventana en sí como un objeto material" (p. 64). Lo que esta afirmación significa es que el espacio arquitectónico es un espacio existencial, "un espacio vivido" y no meramente físico ni estético.

En resumen, el espacio interior de la arquitectura es una experiencia compleja y como tal debe ser apreciada más que como percepción. En ella intervienen las diferentes percepciones visuales, hápticas, auditivas, kinestésica y la memoria como imagen visual que permanece, de lo que se tiene por detrás y no se está viendo ante los ojos; por lo tanto, desde el punto de vista del diseño arquitectónico, es aquél en que el diseñador ha de intervenir con el fin de lograr que la residencia del hombre se complete y mejore en calidad, sea cual fuere su uso. 


\section{Sensaciones espaciales y su influencia en el usuario}

Si se habla de espacio habitable es porque el espacio conceptualizado, diseñado y construido, tiene el carácter de "habitar". En un espacio pensado en el hombre (usuario) que reúne las condiciones ideales del confort -para ser más preciso, del confort psicológico a partir de las características del espacio sociofísico- como esquema cognoscitivo de orientación espacial que acepta información del medio sociofísico y dirige acciones predeterminadas por el cual el individuo puede acceder a condiciones psicofisiológicas favorables que están sujetos a estadios socioculturales y biológicos, determinados por condiciones contextuales, sujetos a juicios de valor (Villalpando \& Barceína, 2012).

Al respecto, Aisa (2012) amplía el concepto y el carácter de "habitar" del espacio creado, citando a Heidegger (1951) quien hace explícita la necesidad de diálogo entre la habitación arquitectónica y el pensar filosófico. El "habitar" es la clave de la unión, al confluir en él tanto el pensar como el construir (p. 16).

En ese sentido, el proceso de diseño, constituye una ciencia multidisciplinar con la intervención de la filosofía unida a la arquitectura.

Al observar el espacio (interior), este devuelve configuraciones espaciales de orden sensorial y espacial, que -a modo de imágenes reproducidas en el cerebro- influyen en el estado emocional y comportamiento en forma positiva o negativa, según la forma, textura y/o color de los elementos que lo estructuran y se presentan. Esta forma conceptual y filosófica de ver y concebir el espacio interior en el diseño arquitectónico, es utilizado poco por los arquitectos de hoy y muchos menos por el estudiante de arquitectura durante su aprendizaje en las escuelas de arquitectura.

Robles \& Esparza (2015) en su investigación sobre "Experiencia perceptiva en el diseño de los espacios interiores", explica esta teoría resumiendo que:

La forma en que percibimos el espacio que habitamos o en el cual nos desarrollamos, se en- cuentra dotada de experiencias que cargan de significado la existencia de todo ser humano. Diseñar estos espacios, es pensar de forma integradora, es adentrarnos en la condición del hombre al habitar y en los componentes que constituyen estos espacios. Delimitar, configurar, organizar, ambientar y embellecer un espacio; pero, sobre todo, generar experiencias perceptivas a través de los sentidos, serán elementos y recursos que determinarán el valor y el significado de cada espacio para ser capaces de generar respuestas cognitivas en el actuar y en la memoria de quienes lo experimentan. (p. 16)

\section{Elementos perceptuales como instrumentos de diseño del espacio (interior vivencial)}

Robles \& Esparza (2015) consideran que la complejidad de la percepción espacial surge a partir del análisis de los recursos perceptivos aplicables en el diseño del espacio interior, los cuales serían: los recursos visuales, táctiles, auditivos, olfativos; añadiendo por último el elemento final: la traducción o reconocimiento por medio del efecto memorial, cuyas características se describen sucintamente a continuación:

\section{Recursos visuales perceptivos}

Trasmisible por medio de la vista y perceptualmente sugerente en el diseño desde los principios básicos como lo son: manejo de la forma y el espacio, orden, armonía, límites, escala, secuencia, etcétera. Ligándose directamente a todo tipo de criterios funcionales y estéticos. Es también reconocido y profundamente analizado en el ámbito de la psicología ambiental y social, por medio del color y el manejo de la luz, ya sea natural o artificial y su implementación en los espacios.

\section{Recursos táctiles perceptivos}

La piel es el órgano más grande del cuerpo por lo cual la sensibilidad del ser humano en relación a lo que toca o simplemente percibe en cuanto al confort del ambiente constituye una experiencia sensorial muy importante en el diseño de los espacios in- 
teriores, ya que se habla de la capa tangible por medio de la cual se pueden generar algunos efectos relacionados con las emociones ligadas a la comodidad o al confort. Elementos como la forma, la densidad y la textura, la solidez, la suavidad, la rugosidad y la temperatura de los materiales (si son cálidos o fríos), lo que es perceptible por medio del tacto.

\section{Recursos auditivos perceptivos}

Este recurso es aplicable a los espacios como instrumento de diseño acústico al proveer al espacio de efectos sonoros o por el contrario limitarlo o absorberlos, para disminuir su audición o magnificarlos, tal como en las áreas de trabajo o de concentración como bibliotecas o en espacios de reproducción y ampliación de sonidos como salas de cine, teatros, etcétera.

\section{Recursos olfativos perceptivos}

El olfato es un sentido de percepción global de estímulo compuesto, esto quiere decir que prioriza olores naturales de los artificiales y otorga características olfativas de tipo negativas y positivas, de tal forma que puede ser aplicable en relación a olores y espacio físico. Dentro de estas características positivas y negativas, se pueden referir aromas que son gratos al olfato y los que generan disgusto; estas características son aplicables como estrategias dentro del diseño del espacio. Un aroma positivo, podría asociarse a frescura en el ambiente y por lo tanto habría una tendencia sobre un olor natural y fresco, vinculado al ambiente y a la circulación de aire. Un ambiente negativo podría ser aquel que provoca disgusto o malestar y se pretendería evitarlo generando flujos de viento cruzado.

\section{Efecto memorial}

La interacción constante entre el espacio, el hombre y por lo tanto sus sentidos, otorgan experiencias completas, conexiones sublimes que incluso Gastón Bachelard (1983) supone e identifica como una experiencia del ensueño. Un espacio arquitectónico que puede encuadrar, fortalecer y concen- trar todos los pensamientos; es así, que la trascendencia de la experiencia perceptiva del espacio conduce al resultado de un efecto memorial al recuerdo, a la evocación de lo ya acontecido; provocando añoranzas, nostalgias, alegrías, etcétera, es decir, emociones.

Los mismos autores a modo de conclusión, consideran académicamente que el diseño del espacio interior atribuye competencias de intenciones respecto a los sentidos y su estimulación potencializando el éxito. Los espacios en relación a las características de las personas que los experimentarán, requieren del establecimiento de estrategias didácticas y de experimentación, que permitan abarcar todos los elementos perceptuales y su repercusión como efecto memorial y psicológico. De tal forma que el espacio no se limita al elemento visual, la belleza del inmueble, la distinción de las formas por medio de los ojos, sino también a la presencia de elementos más complejos de ser aplicables en el diseño. Generar una propuesta de diseño es contribuir a la generación de experiencias perceptivas, un diseño experiencial que se funda en una única dimensión, creando así no solo espacios compositivamente funcionales y estéticos; sino también, la construcción de vivencias y sensaciones adecuadas para cada tipo de usuario y ambiente.

\section{Algunas experiencias en el diseño del espacio interior arquitectónico y su influencia en el usuario}

En el 2003, nació la Academia de la Neurociencia para la Arquitectura en San Diego, en donde expertos en neurociencia y arquitectura, establecen sinergias para entender y conocer cómo el entorno modula el cerebro; de este modo, si los diseños arquitectónicos incorporan principios neurológicos, seguramente potenciarán la creatividad y el confort de quienes ocupen esos edificios -en especial sus espacios interiores- donde el usuario participa de las sensaciones emotivas que le producen la estructura espacial de los mismos, mediante las experiencias perceptuales.

Al respecto, la Organización Mundial de la Salud (OMS) (1982), habla de edificios enfer- 